\title{
Ensino e Aprendizagem em Estágio Supervisionado: Estágio Integrado em Saúde
}

\author{
Teaching and Learning in Supervised \\ Internship: Integrated Internship in Health
}

\author{
Emanuelle Cavalcante Pimentel \\ Maria Viviane Lisboa de Vasconcelos ${ }^{I}$ \\ Renato Santos Rodarte ${ }^{I}$ \\ Célia Maria Silva Pedrosa ${ }^{I}$ \\ Fernando Silvio Cavalcante Pimentel
}

\section{PALAVRAS-CHAVE \\ - Estágio; \\ - Ensino; \\ - Aprendizagem; \\ - Educação em Saúde.}

\section{RESUMO}

As Instituições de saúde de ensino superior têm buscado efetuar mudanças em seus projetos politico-pedagógicos, replanejando, assim, seus currículos. Esta pesquisa teve por objetivo avaliar o processo de ensino-aprendizagem no estágio supervisionado, quando realizado com a integração de estudantes de diversos cursos na área da saúde, utilizando como abordagem um estudo de caso etnográfico, por meio de observação participante, questionário semiestruturado e grupo focal. Mediante a triangulação dos dados, pôde-se perceber a importância da interdisciplinaridade, do trabalho em equipe, da contribuição para a formação profissional e do papel do docente como facilitador do processo de ensino-aprendizagem, estimulando o senso crítico e a tomada de decisão. A proposta do Estágio Integrado permite um novo olhar no processo de ensino-aprendizagem, aliada à diversificação do cenário de prática, principalmente quando se fala em ensino na saúde. O Estágio Integrado pode ser entendido como uma estratégia pedagógica para tentar superar a fragmentação do conhecimento e ser utilizado como um referencial por outras Instituições de ensino na saúde que buscam formar profissionais de saúde com perfil humanista, capazes de atuar na integralidade da atenção à saúde e em equipe.

\begin{abstract}
Higher education health institutions have sought to make changes to their political-pedagogical projects, thus redesigning their curricula. This study aimed to evaluate the teaching-learning process in supervised internships that integrate students from diverse health care courses, using the approach of an ethnographic case study through participant observation, semi-structured questionnaire and focus groups. Triangulated data indicates the importance of interdisciplinary structure, teamwork, the teacher's role and contribution as facilitator of the training process, encouraging critical thinking and decision making. The proposed Integrated Internship allows a new vision of the teaching-learning process, coupled with diversified practical situations, particularly as regards education in health. It can be understood as a pedagogical strategy aimed at overcoming knowledge fragmentation and can be used as a benchmark by other educational institutions in health, seeking to train health professionals with a humanistic profile, ready to work in comprehensive health care and as part of a team.
\end{abstract}




\section{INTRODUÇÃO}

No Brasil, a formação profissional voltada às necessidades sociais vem suscitando profundas mudanças na educação superior e de modo particular na formação superior em saúde.

Atualmente, o desafio das Instituições de Ensino Superior (IES) é formar profissionais de saúde com perfil humanista, capazes de atuar na integralidade da atenção à saúde e em equipe, características indispensáveis aos serviços do Sistema Único de Saúde (SUS), considerando também as Diretrizes Curriculares Nacionais (DCN) ${ }^{1}$.

Nesse contexto, as IES têm buscado efetuar mudanças em seus projetos político-pedagógicos no sentido de reformular seus currículos ${ }^{2}$.

A diversificação dos cenários de prática é compreendida como uma das estratégias para a transformação curricular ${ }^{3}$. Essa estratégia aproxima os estudantes da vida cotidiana da população e desenvolve um olhar crítico, possibilitando cuidar dos reais problemas da sociedade.

O estágio curricular, sob supervisão docente, deverá ser desenvolvido de forma articulada e com complexidade crescente ao longo do processo de formação ${ }^{4}$. Assim, a diversificação de cenários de prática deve estar presente ao longo de toda a formação, permitindo que os estudantes tenham a oportunidade de aprender e trabalhar em todos os espaços em que se dá a atenção à saúde 5 .

Em 2009, o Centro Universitário Cesmac, em Maceió (AL), em conformidade com os princípios do SUS e das DCN, implementou o Estágio Integrado em Saúde (EI), com o objetivo de integrar os alunos das Clínicas dos cursos de saúde (Fisioterapia, Nutrição, Enfermagem, Farmácia, Odontologia e Biomedicina).

O Estágio Integrado possibilita aos acadêmicos da área de saúde uma prática multidisciplinar com enfoque na vigilância à saúde, por meio do diagnóstico dos principais problemas de saúde da comunidade atendida. Além da assistência à comunidade, os estudantes desenvolvem senso crítico e aprendem a tomar decisões em equipe, atendendo a uma área que abrange 332 famílias.

A oferta destes espaços de aprendizagem aos alunos remete ao que diz Morin ${ }^{6}$, quando afirma que a reforma da universidade promove um paradoxo: não se pode reformar as instituições se antes as mentes não forem reformadas, mas só se pode reformar as mentes se as instituições forem previamente reformadas.

Nesse sentido, espera-se que a função docente dê sustentabilidade às atividades educativas que se iniciam com a familiarização do estudante com o novo processo pedagógico que passa a vivenciar ${ }^{7}$.
Neste processo de aprendizagem, exercem papel fundamental metodologias ativas de ensino-aprendizagem que viabilizem a construção dos conhecimentos com base nos problemas da realidade, na integração de conteúdos básicos e profissionalizantes, entre teoria e prática, e também na produção de conhecimento integrada à docência e à atenção ${ }^{1}$. $\mathrm{O}$ desafio para o professor é romper com os limites de sua formação fragmentada e reconstruir relações com outras áreas de conhecimento $^{8}$.

Esta pesquisa, por meio de metodologia qualitativa e com uso da triangulação, teve como objetivo avaliar o processo de ensino-aprendizagem no Estágio Integrado em saúde.

\section{Concepções do estágio em saúde}

Conforme o parecer n⿳⼈ㅡㄹ 21, de 2001 do Conselho Nacional de Educação, o estágio curricular na área de saúde permite, de maneira singular, uma reflexão sobre as atividades da futura profissão, possibilitando um processo de ensino-aprendizagem realizado por meio da prática neste exercício. Proporciona ainda ao aluno a vivência e o relacionamento com outros profissionais de saúde ${ }^{9,10}$.

A articulação entre ensino e serviço, segundo Campos et al. ${ }^{11}$, apresenta-se como uma ferramenta para efetiva integração entre teoria e prática, pois estimula uma reflexão acerca da realidade, possibilitando ao aluno elaborar críticas e buscar soluções adequadas para os problemas de saúde encontrados, com compromisso e responsabilidade com o usuário.

Garcia ${ }^{5}$ ressalta que o processo ensino-aprendizagem nos campos de estágio apresenta aspectos diferenciados daqueles efetuados em salas de aula, permitindo que as relações se estendam além da docente-discente, incluindo também os usuários e a equipe de trabalho.

\section{MÉTODOS}

A coleta dos dados ocorreu de abril a novembro de 2012 junto aos docentes e alunos do Estágio Integrado do Centro Universitário Cesmac, em Maceió (AL).

A pesquisa usou como abordagem o estudo de caso do tipo etnográfico ${ }^{12}$. Os dados foram construídos por meio de observação participante, questionário semiestruturado e grupo focal. A associação entre o grupo focal e a observação participante permitiu comparar o conteúdo produzido no grupo com o cotidiano dos participantes em seu ambiente natural, além de correlacioná-lo com os dados dos questionários.

\section{Observação participante}

Durante a técnica de observação participante, o pesquisador fez um registro detalhado das atividades e do conteúdo das 
observações em um diário de campo (descrição dos fatos, falas, comportamento, ações e conversas). Na parte reflexiva, ele registrou comentários pessoais, dúvidas, expectativas, opiniões e reflexões metodológicas.

Foram realizados 35 registros das observações. O pesquisador fez uso apenas do seu registro do diário de campo, não havendo acesso a nenhum registro dos docentes do Estágio Integrado.

Os dados levantados foram submetidos a análise de conteúdo, que, em conformidade com $\operatorname{Bardin}^{13}$, se caracteriza como um conjunto de técnicas de análise das comunicações a fim de obter, por procedimentos sistemáticos e objetivos de descrição do conteúdo das mensagens, indicadores (quantitativos ou não) que permitam inferir conhecimentos relativos às condições de produção/percepção (variáveis deduzidas) destas mensagens.

\section{Questionário semiestruturado}

O questionário semiestruturado foi utilizado com o objetivo de verificar a percepção do discente em relação ao processo de ensino-aprendizagem do Estágio Integrado

Foram aplicados 64 questionários. Tendo em vista que algumas respostas começaram a se repetir, optou-se pelo uso da saturação ${ }^{14}$. O fechamento amostral por saturação teórica é operacionalmente definido como a suspensão da inclusão de novos participantes quando os dados obtidos passam a apresentar, na avaliação do pesquisador, certa redundância ou repetição ${ }^{15}$.

\section{Grupo focal}

O grupo focal tem sido utilizado internacionalmente na estruturação de ações diagnósticas e levantamento de problemas, bem como no planejamento de atividades educativas, como objeto de promoção em saúde e meio ambiente. Pode ser empregado também para revisão do processo de ensino-aprendizagem ${ }^{16}$.

Participaram do grupo focal dez professores que fazem parte do Estágio Integrado, o docente mediador, o docente pesquisador e dois professores convidados, para registro das falas.

\section{RESULTADOS}

Este estudo avaliou o processo ensino-aprendizagem em estágio supervisionado que integrou alunos das diversas áreas de saúde, chamado de Estágio Integrado, na tentativa de verificar o fazer pedagógico relacionado ao trabalho em saúde, que tem como foco o uso da problematização do cotidiano. Para melhor descrição dos resultados, optou-se pelo método da triangulação e foram utilizados codificação para o registro das falas: OD: observação participante - docente; OA: observação participante - aluno; QA: questionário - aluno; FD: grupo focal - docente
O questionário semiestruturado foi respondido por 64 alunos, que fazem parte dos cursos de Nutrição, Fisioterapia, Farmácia, Odontologia e Biomedicina (Tabela 1).

\begin{tabular}{|c|c|c|}
\hline \multicolumn{3}{|c|}{ TABELA 1} \\
\hline Cursos & $\mathbf{N}$ & $\%$ \\
\hline Farmácia & 22 & 35,7 \\
\hline Fisioterapia & 06 & 9,37 \\
\hline Odontologia & 09 & 14,6 \\
\hline Nutrição & 16 & 25,0 \\
\hline Biomedicina & 05 & 7,8 \\
\hline Enfermagem & 06 & 9,37 \\
\hline Total & 64 & 100 \\
\hline
\end{tabular}

Fonte: Dados da pesquisa.

Os alunos apresentam idade média de 22 anos, sendo 49 (76,5\%) do gênero feminino e 15 (23,4\%) do gênero masculino.

Os objetivos do Estágio Integrado e as Diretrizes do SUS são conhecidos por $54(84,4 \%)$ alunos. No entanto, 10 (15,6\%) responderam que a integralidade da atenção seria o acesso à saúde em todos os níveis.

Em relação ao questionamento sobre interdisciplinaridade, $46(71,8 \%)$ alunos responderam que sabiam o que seria interdisciplinaridade, enquanto $18(28,2 \%)$ responderam que não sabiam.

A percepção do docente sobre interdisciplinaridade registrou-se por meio de uma atividade com crianças de uma creche, com o tema "lavagem das mãos", em que os alunos de diversos cursos da saúde fizeram uma paródia e ensinaram as crianças a lavagem correta das mãos. Posteriormente, um docente fez um comentário sobre a satisfação com o resultado do trabalho interdisciplinar:

"[...] gente, fiquei muito feliz... fui lavar as mãos e vi três crianças lavando as mãos como foi ensinado na semana passada... é muito legal quando verificamos que o que foi passado a eles foi percebido, aprendido... (OD1)"

Esta interdisciplinaridade também foi citada no grupo focal:

\footnotetext{
"Vislumbra a interdisciplinaridade, compreender os elos em comum nas diferentes graduações. Promoção e prevenção. Os alunos conhecem os outros cursos, o que cada curso faz. Treinamento em conjunto, enfatizando o objetivo do SUS. Torna-se sensível ao ver o paciente, com a particularidade de cada um. No momento em que precisa, usa-se a especialida$d e^{\prime \prime} .(F D 7)$
} 
A integração entre os alunos dos diversos cursos no Estágio Integrado é marcante. Noventa por cento deles relataram que a integração é muito boa, surgindo alguns registros de possibilidade de diálogo entre as profissões, troca de informações e discussão do tema saúde, como pode ser observado na fala de um estudante:

"[...] aprendi que cada um é importante na sua área de atuação e que juntos podemos fazer a diferença..." (QA3)

Uma dificuldade percebida por alguns alunos quanto à integração está relacionada ao tempo de convivência entre eles, visto que esse tempo é desigual. O momento integrado vivenciado foi inserido em disciplinas de estágio que já existiam nas matrizes dos cursos, havendo para cada curso uma carga horária diferente.

Metade dos alunos relatou que se identificou com todas as atividades do Estágio Integrado e que colocou em prática o que aprendeu na faculdade e no Estágio integrado. Essa observação surge na fala de uma aluna:

"[...] quando uma atividade pela qual meu grupo foi responsável foi realizada com êxito, havendo contribuição de todos os alunos, com o conhecimento que cada um possui, adquirido em seu curso, foi muito legal, foi a que mais gostei..." (QA4)

A dificuldade surge quando uma atividade não está relacionada diretamente a algum curso, e os alunos tendem a se dispersar, sendo necessária a intervenção do professor. Esta atitude ainda está relacionada à cultura do trabalho isolado por disciplina. Essa resistência é observada também no relato do professor no grupo focal:

"Mudança da concepção do aluno no convívio em equipe; é muito forte, eles não conseguem entender a proximidade. Muito resistente. Invasão de território..." (FD8)

O Estágio integrado é considerado importante para a vida profissional dos alunos nos seguintes aspectos: valorização e importância do trabalho em equipe; integração com outros profissionais; humanização da atenção à saúde; conhecimento e reconhecimento das outras áreas da saúde - o que pode ser observado na resposta de uma aluna:

"[...] a partir deste estágio, pude perceber a importância do trabalho em conjunto e como devemos nos comportar no serviço. Por isso, pretendo ser uma profissional dedicada e fazer o melhor para os meus pacientes, tratando-os primeiramente com muito amor..." (QA5)
E na fala de uma docente:

"O Estágio integrado permitiu uma quebra de paradigmas para os cursos que não participam da atenção básica. Hoje isso mudou: os profissionais participando ativamente, mostrando aos alunos o trabalho de forma integral. Conhece melhor o outro profissional". (FD4)

O docente tem um papel fundamental, e durante o Estágio Integrado sua contribuição foi referida pelos alunos como: boa, positiva, sempre presente, auxiliar, integração, incentivo, segurança, trabalho com o SUS, treinamento e orientação, esta última com $12 \%$ de registro. Mas, para os docentes da área da saúde, este papel de facilitador não é tão fácil, como cita uma professora durante o grupo focal:

"O profissional da saúde não é formado para ser docente. Este é o grande desafio. Tem que acreditar na proposta". (FD8)

A percepção do docente sobre o Estágio Integrado influi fortemente sobre uma nova forma de pensar e agir em saúde e no processo de ensino-aprendizagem. Essa percepção fica clara no grupo focal quando um docente diz que:

“Em 2009, em virtude da necessidade, angústia de alguns docentes, eles tentaram integrar as áreas de saúde para promover a integralidade da atenção. Atender de forma integral". (FD3)

E traz uma reflexão mais aprofundada na fala de outra docente:

"Refletir sobre as necessidades do paciente, com uma reflexão mais crítica: compromisso integral com a saúde, de acordo com as Diretrizes Curriculares. Articulação da teoria e da prática". (FD3)

Alguns obstáculos registrados na observação participante e no relato dos docentes no grupo focal apontam a necessidade de um Estágio Integrado com fluxo contínuo em benefício do usuário, o que não ocorre no período de recesso escolar.

A observação da avaliação do aluno no processo de ensino-aprendizagem aponta falta de sistematização da mesma. São utilizadas várias formas de avaliar inovadoras, como o portfólio e o checklist, que, no entanto, são realizadas separadamente por cada grupo de alunos dos vários cursos. Emerge dessas observações a necessidade de uma avaliação integrada que realmente avalie o objetivo do aprendizado do aluno no Estágio Integrado, um processo de avaliação elaborado em conjunto pelo grupo dos diversos docentes e aplicada integralmente. 


\section{DISCUSSÃO}

\section{Processo de ensino-aprendizagem}

Os resultados apontaram o quanto é válida a tentativa de integração entre os alunos dos diversos cursos da área da saúde. Essa integração contribui para o ensino-aprendizagem em termos de crescimento e desenvolvimento de uma pessoa em sua totalidade, abarcando minimamente quatro grandes áreas: conhecimento; afetivo-emocional; habilidades e atitudes ou valores ${ }^{17}$.

É em meio a este universo que os docentes do Estágio Integrado passam aos alunos a importância de fazer saúde pública e trabalhar a integralidade da atenção numa equipe multiprofissional.

De acordo com a Lei 8.080 de 19 de setembro de 1990:

[...] integralidade de assistência é entendida como um conjunto articulado e contínuo das ações e serviços preventivos e curativos, individuais e coletivos, exigidos para cada caso em todos os niveis de complexidade do sistema. $(p .3)^{18}$

Araújo $^{19}$ registra que, para o desenvolvimento de ações de saúde na perspectiva da integralidade, é necessária uma aproximação integral entre os sujeitos que prestam o cuidado. Ou seja, estabelecer uma prática de comunicação entre os profissionais de saúde como estratégia para o enfrentamento dos conflitos, rompendo com as antigas estruturas hierarquizadas, tão presentes no modelo de saúde hegemônico.

A proposta do Estágio Integrado nos remete ao que dizem Carvalho e Ceccim²0: para ser um profissional de saúde, há necessidade de conhecimento científico e tecnológico, mas também de conhecimento de natureza humanística e social relativo ao processo de cuidar, sendo preciso desenvolver projetos terapêuticos singulares, formular e avaliar políticas, além de coordenar e conduzir sistemas e serviços de saúde.

A escolha de métodos de ensino-aprendizagem atrelados a uma nova forma de interpretar e agir em saúde, a diversificação de cenários, a capacitação dos docentes que serão multiplicadores do processo e o estímulo à produção do conhecimento na área de saúde individual e coletiva são peças fundamentais para a criação de cenários propícios à aprendizagem e à organização do serviço de saúde ${ }^{21}$.

Quando a integração ensino-serviço acontece de forma efetiva, unindo docentes, estudantes e profissionais de saúde com foco central no usuário, esta relação entre o ensino e a produção dos cuidados em saúde se ameniza ${ }^{22}$.

Para isso, é necessário também o trabalho em equipe, que tem como meta a obtenção de elementos sobre os diferentes fatores que interferem no processo saúde-doença. A ação in- terdisciplinar possibilita a prática de o profissional se reconstruir na prática do outro, ambos sendo transformados para a intervenção na realidade em que estão inseridos ${ }^{19}$.

\section{Interdisciplinaridade}

A interdisciplinaridade no estágio está presente nas ações do grupo integrado. Podemos comparar com o que diz Japiassu ${ }^{23}$ quando refere que a interdisciplinaridade se caracteriza pela intensidade das trocas entre as especialidades e pelo grau de integração real das disciplinas.

A construção da interdisciplinaridade tem sido um processo contínuo e crescente no setor saúde, na tentativa de superar a fragmentação do conhecimento humano, em busca de uma visão globalizada da complexa dimensão do processo saúde-doença. A ação interdisciplinar pressupõe a possibilidade da prática de um profissional se reconstruir na prática do outro, ambos sendo transformados para a interferência na realidade em que estão inseridos ${ }^{24,19}$.

No trabalho em equipe, o diálogo assume uma importância significativa e indispensável ao bom desenvolvimento da atividade. Isto aponta à possibilidade de superação da prática comunicativa. Assim, o trabalho em equipe "provoca" a escuta do outro, o que pressupõe o estabelecimento de uma via de comunicação ${ }^{19}$.

Para Albuquerque et al. ${ }^{22}$, os espaços onde se dá o diálogo entre o trabalho e a educação assumem lugar privilegiado para a percepção que o estudante vai desenvolvendo acerca do outro no cotidiano do cuidado. São espaços de cidadania, onde profissionais do serviço e docentes, usuários e o próprio estudante vão estabelecendo seus papéis sociais na confluência de seus saberes, modos de ser e de ver o mundo.

A aproximação do cotidiano pode permitir tornar a educação significativa por meio da vivência de situações. Assim, o processo ensino-aprendizagem em estágios que busque rever estes modelos se diferencia fundamentalmente da educação formal, dita escolarizada, pois a integração ensino/trabalho não se limita ao processo dado institucionalmente, mas se impõe no cotidiano, nas relações entre sujeitos e na comunicação/interação de seus projetos ${ }^{5}$.

\section{Contribuição do docente}

No Estágio Integrado, o docente se comporta como mediador/facilitador do processo de ensino-aprendizagem. Muitas vezes, isto exige que ele assuma uma nova postura, uma atitude diferente de sua formação fragmentada, o que demanda superação a cada atividade não desenvolvida como planejado. Este docente supera as dificuldades pela força de vontade, pela sintonia da equipe. Existe uma motivação da parte dele, 
uma vontade de dar certo, o que exige dele uma mudança de postura frente ao aluno.

Pimentel ${ }^{25}$ sugere que o educador é o sustentáculo do processo de ensino-aprendizagem e que ele precisa conhecer em profundidade e extensão, ter experiência, vontade e gosto por aquilo que faz, bem como saber educar: aproximar-se, ouvir, respeitar, interagir e, principalmente, dialogar.

Assim, o novo papel docente exigiria do professor assumir o ensino-aprendizagem como mediação da aprendizagem ativa do estudante com o auxílio pedagógico do professor, transformando a escola num cenário com práticas inter e transdisciplinares e integradas à vida cotidiana, conhecendo e aplicando estratégias e metodologias ativas de ensinar-aprender a pensar, a aprender, a cuidar e avaliar ${ }^{26}$.

As estratégias utilizadas no Estágio Integrado são muitas, sempre com criatividade e organização pedagógica, mediante um cronograma de atividades, sequência das aulas e do método, oficinas, portfólio e treinamentos.

Ampliam-se as relações entre aprender, ensinar e avaliar, com alunos e professores, em diferentes patamares, trocando saberes e objetivos. É um grande desafio para os professores vindos de uma formação acadêmica marcada pela transmissão de informações. Desafio que demanda movimentação e disponibilidade para aprender o novo, assumindo lugar de orientação, facilitação e escuta dos alunos, com uma função mediadora ${ }^{27}$.

Nessa perspectiva, o professor tem um papel extremamente importante como mediador entre o aluno e o conhecimento, facilitando, incentivando e motivando a aprendizagem ao desenvolver um conteúdo de forma a permitir que o estudante colete, relacione, organize, manipule, discuta e debata as informações com seus colegas e com o professor, produzindo um conhecimento significativo que se incorpore ao seu mundo, possibilitando que o aluno compreenda a realidade humana e social onde está inserido e na qual pode interferir, proporcionando uma educação transformadora ${ }^{28}$.

A proposta do Estágio Integrado surge, então, como um referencial para outras Instituições de ensino na saúde que buscam atender às Diretrizes Curriculares Nacionais, ao SUS, ao cuidado com o próximo e à interdisciplinaridade.

\section{CONCLUSÃO}

O Estágio Integrado do Centro Universitário Cesmac, em Maceió (AL), pode ser entendido como uma estratégia pedagógica encontrada por um grupo docente para tentar superar a fragmentação do conhecimento por meio da atenção integral, estimulando o senso crítico e a tomada de decisão.

A diversificação do cenário de prática, principalmente quando se fala em ensino na saúde, permite ao aluno uma vivência bem próxima da que ele irá encontrar na sua vida profissional.

Para os docentes envolvidos no Estágio Integrado, o processo de ensino-aprendizagem está atrelado à forma de pensar em saúde, por meio da integração, do compromisso com a atenção integral do indivíduo, do trabalho em equipe e das ações interdisciplinares, baseadas nos princípios do SUS.

A proposta do Estágio Integrado é um grande desafio para os alunos, que trazem uma experiência de disciplinas fragmentadas, voltadas às especialidades de cada área de atuação, e para os docentes, que, no caso do Estágio Integrado, não tiveram formação pedagógica.

A incorporação do Estágio Integrado à matriz curricular das instituições de ensino superior em uma disciplina específica - como, por exemplo, Saúde Coletiva, com a mesma carga horária e período de cada curso, com sistema de avaliação integrado - é uma sugestão para viabilizar a real integração entre alunos e docentes dos diversos cursos. Tudo isso atrelado à capacitação docente.

\section{AGRADECIMENTOS}

Ao Centro Universitário Cesmac, Maceió Alagoas, Brasil, local da coleta dos dados. A Anne Laura Costa Ferreira.

\section{REFERÊNCIAS}

1. Feuerwerker L. Educação dos profissionais de saúde hoje: problemas, desafios, perpectivas, e as propostas do Ministério da saúde. Revista da ABENO, São Paulo. 2003; 3(1):24-27.

2. Batista NA, Batista SH, Abdalla IG, org. Ensino em Saúde: visitando conceitos e práticas. São Paulo: Arte \& Ciência; 2005. p.39-54.

3. Ferreira RC, Silva RF, Agner CB. Formação do Profissional Médico: a Aprendizagem da Atenção Básica de Saúde. Revista Brasileira de Educação Médica. 2007;31(1):52-59.

4. Brasil. Ministério da Educação. Conselho Nacional de Educação. Parecer no CNE/CES 1.133/2001. Diretrizes Curriculares Nacionais dos Cursos de Graduação em Enfermagem. Medicina e Nutrição. Brasília, DF, ago; 2001.

5. Garcia MAA. Knowledge, action and education: teaching and learning at healthcare centers. Interface _ Comunic, Saúde, Educ 2001;5(8):89-100.

6. Morin E, Almeida MC, Carvalaho E. Educação e Complexidade: os sete saberes e outros ensaios. 3.ed. São Paulo: Cortez; 2005.

7. Ferreira RC, Fiorini VML, Crivelaro E. Formação profissional no SUS: o papel da Atenção Básica em Saúde na perspectiva docente. Revista Brasileira de Educação Medica. 2010; 34(2). 
8. Rossoni E, Lampert J. Formação de profissionais para o Sistema Único de Saúde e as diretrizes curriculares. Boletim da Saúde. 2004; 18(1):87-98.

9. Brasil. Conselho Nacional de Educação. Parecer CNE/CP 21/2001.

10. Magalhães LM. Estágio Supervisionado e metodologia do ensino em uma abordagem dialética. Anais do 5. Seminário de Estágio Supervisionado do Curso de Pedagogia. Anapólis: Fundação Universidade estadual de Goiás. 2007;3; 2009.

11. Campos FE, Ferreira JRF, Feuerwerker L, Sena RR, Campos JJB, Cordeiro H, Luís Cordoni L. Caminhos para aproximar a formação de profissionais de saúde das necessidades da atenção básica. Revista Brasileira de Educação Médica. 2001;25(2).

12. André MEDA. Etnografia da prática escolar. Campinas: Papirus; 2005.

13. Bardin L. Análise de conteúdo. 70 ed. Lisboa; 1997. 226p.

14. Bogdan R, Biklen SK. Qualitative research for educacion: an introduction to theory and methods. Boston: Allynand Bacon; 1982.

15. Fontanela BJB, Ricas J, Turato ER. Amostragem por saturação em pesquisas qualitativas em saúde: contribuições teóricas. Rio de Janeiro: Cadernos de Saúde Pública. 2008;24.

16. Lervolino SA, Pelicioni MCF. A utilização do grupo focal como metodologia qualitativa na promoção da saúde. Revista da Escola de Enfermagem da Universidade de São Paulo 2001;35(2):115-21.

17. Masseto MT. Competência Pedagógica do Professor Universitário. São Paulo: Summus; 2003.

18. Brasil. Lei 8080 promulgada em 19 de setembro de 1990. Brasília, DF: Senado Federal.

19. Araújo MBS, Rocha MP. Trabalho em equipe: um desafio para a consolidação da estratégia de saúde da família. Ciência \& Saúde Coletiva 2007; 12(2): 455-464.

20. Carvalho YM, Ceccim RB. Formação e Educação em Saúde: aprendizados com a saúde coletiva. In: Campos GWS, Minayo MCS, Akermam M, Drumond M, Carvalho YM. Tratado de saúde coletiva. Rio de Janeiro: Hucitec; Fiocruz; 2006. p.149-182.

21. Mattos D. As novas diretrizes curriculares e a Integralidade em saúde: uma análise das possíveis contribuições da odontologia para o trabalho em equipe. Rio de Janeiro; 2006. Doutorado [Tese] — Universidade Estadual do Rio de Janeiro.

22. Albuquerque VS, Gomes AP, Rezende CHA, Sampaio MX, Dias OV, Lugarinh RM. A Integração Ensino-serviço no
Contexto dos Processos de Mudança na Formação Superior dos Profissionais da Saúde. Revista Brasileira de Educação Médica 2008; 32(3):356-362.

23. Japiassu H. Interdisciplinaridade e patologia do saber. Rio de Janeiro: Imago; 1976.

24. Garrutti EA, Santos SR. A interdisciplinaridade como forma de superar a fragmentação do conhecimento. Revista de Iniciação Científica da FFC 2004;4(2).

25. Pimentel MG. O professor em construção. Campinas: Papirus; 1993.

26. Komatsu RS. Educação Médica: Responsabilidade Social de Quem? Em Busca dos Sujeitos da Educação do Novo Século. Revista Brasileira de Educação Medica; 2002;26(1):55-61.

27. Batista N, Batista SH, Goldenberg P, Seiffert O, Sonzogno MC. O enfoque problematizador na formação de profissionais da saúde. Revista de Saúde Pública.2005;39(2):231-237.

28. Gasparin JL. Uma didática para a pedagogia histórico-crítica. 3. ed. rev. Campinas: Autores Associados; 2005. (Coleção educação contemporânea)

\section{ÉTICA}

Atendendo à Resolução nº 196/96 do Conselho Nacional de Saúde, esta pesquisa foi encaminhada para análise do Comitê de Ética do Centro Universitário Cesmac, em Maceió (AL), tendo obtido aprovação em 30/05/12, sob o Protocolo nº 1311/12.

\section{CONTRIBUIÇÃO DOS AUTORES}

Emanuelle Cavalcante Pimentel: coleta, análise e interpretação dos dados, discussão e proposição teórica. Maria Viviane Lisboa de Vasconcelos: orientação da pesquisa e análise dos dados. Renato Santos Rodarte: co-orientação da pesquisa e análise dos dados. Célia Maria Silva Pedrosa: revisão metodológica. Fernando Silvio Cavalcante Pimentel: coleta de dados e revisão teórico-metodológica.

\section{CONFLITO DE INTERESSES}

Os autores declaram não haver conflito de interesses.

\section{ENDEREÇO PARA CORRESPONDÊNCIA}

Emanuelle Cavalcante Pimentel

Rua Dr. Paulo Neto, 138

Trapiche da Barra - Maceió

CEP 57072-970 - AL

E-mail: emanuellecpimentel@hotmail.com 